\title{
Micro-computed tomographic evaluation of the effect of fluoride agents on white spot lesions: An in vitro study
}

\author{
Sook-Chan Hong ${ }^{\mathrm{a}}$ (1) \\ Dong-Yul Lee ${ }^{\mathrm{b}}$ \\ Yae-Jin Kim ${ }^{\mathrm{c}}$ (1)
}

${ }^{a}$ Major in Dentistry, Department of Medicine, Graduate School of Korea University, Seoul, Korea

${ }^{b}$ Department of Dentistry, College of Medicine, Korea University, Seoul, Korea

'Department of Orthodontics, Korea University Guro Hospital, Seoul, Korea
Objective: To investigate remineralizing effect of three fluoride regimens on artificially demineralized enamel around orthodontic bracket by analyzing mineral density (MD) acquired from micro-computed tomography (micro-CT). Methods: Forty-eight bracket bonded bovine incisors were prepared to create demineralized enamel (DE) surface. The samples were divided into four groups according to the fluoride regimen: 1) no fluoridation, 2) 1.23\% acidulated phosphate fluoride (APF) gel, 3) fluoridated toothpaste, and 4) 0.05\% sodium fluoride mouthwash. Micro-CT was scanned after demineralization (T0), and 2 weeks (T1) and 4 weeks (T2) of fluoridation. Results: APF gel showed highest remineralization of DE during T1-T0 interval among the groups $(p<$ 0.05); followed by toothpaste, mouthwash and no fluoridation. APF gel and toothpaste demonstrated significant increase in MD after 4 weeks of application $(p<0.05)$. Conclusions: Remineralization effects of three fluoride regimens were depicted through micro-CT analysis, of which APF gel was most effective. [Korean J Orthod 2022;52(1):75-79]

Key words: Cariology, Fluoride, Micro-computed tomography
Received June 21, 2021; Accepted July 19, 2021.

Corresponding author: Yae-Jin Kim.

Clinical Assistant Professor, Department of Orthodontics, Korea University Guro Hospital, 148 Gurodong-ro, Guro-gu, Seoul 08308, Korea.

Tel +82-2-2626-1524 e-mail estelle7@korea.ac.kr

How to cite this article: Hong SC, Lee DY, Kim YJ. Micro-computed tomographic evaluation of the effect of fluoride agents on white spot lesions: An in vitro study. Korean J Orthod 2022;52:75-79.

(C) 2022 The Korean Association of Orthodontists.

This is an Open Access article distributed under the terms of the Creative Commons Attribution Non-Commercial License (http://creativecommons.org/licenses/by-nc/4.0) which permits unrestricted non-commercial use, distribution, and reproduction in any medium, provided the original work is properly cited. 


\section{INTRODUCTION}

Fixed orthodontic appliances are a well-known cause for the increased incidence of white spot lesions (WSLs), which result from subsurface enamel demineralization. ${ }^{1,2}$ Despite numerous efforts to prevent the formation of WSLs, one study showed that up to $97 \%$ patients treated with fixed appliances developed WSLs of varying degrees after therapy. ${ }^{3}$ Interventions such as the application of casein phosphopeptide amorphous calcium phosphate (CPP-ACP) complexes, resin infiltration, and microabrasion have shown to be effective in alleviating incipient carious lesions; however, the gold standard treatment for WSLs remains to be established. ${ }^{4-7}$

Although multiple studies have reported that topical fluoride is an effective cariostatic agent with the ability to induce remineralization of initial carious lesions, ${ }^{8-10}$ studies analyzing the remineralization effect of different fluoride regimens on WSLs by comparing the changes in the mineral density (MD) are scarce. Therefore, this in vitro study aimed to investigate the remineralization effects of three topical fluoride regimens on artificially induced WSLs around orthodontic brackets bonded to bovine teeth using micro-computed tomography (microCT).

\section{MATERIALS AND METHODS}

\section{Sample preparation}

Forty-eight bovine incisors with sound enamel (SE) surfaces were selected. A maxillary first incisor bracket (0.018-inch slot standard, Archist; Daeseung, Seoul, Korea) was bonded to the enamel surface using Transbond XT (3M Unitek, Monrovia, CA, USA). The surface was divided along the line of the bracket slot into demineralized enamel (DE), which was subjected to artificial demineralization, and SE, which was covered with acidresistant nail varnish.

The demineralizing solution was prepared with distilled water and $1.0 \mathrm{M}$ lactic acid, calcium phosphate tribasic saturated at 50\% concentration, and 1\% Carbopol (\#980; Lubrizol Co., Wickliffe, OH, USA) adjusted to $0.2 \%$ of the total volume. The $\mathrm{pH}$ was adjusted to 4.5 by adding $50 \% \mathrm{NaOH}$ solution. The samples were immersed in the demineralizing solution and stored in an incubator at $37^{\circ} \mathrm{C}$ for 96 hours. The demineralizing solution was replaced every 24 hours. The samples were cleansed with distilled water, following the demineralization procedure.

The samples were randomly divided into four groups ( $n=12$ ) according to the fluoride treatment. Group 1 was the control, and the samples were only immersed in artificial saliva (Xerova Solution; Kolmar, Seoul, Korea) without fluoridation. Group 2 underwent application of $1.23 \%$ acidulated phosphate fluoride (APF) gel (lonite
APF Thixotropic gel; Dharma, Miami, FL, USA) for 4 minutes once a week. Fluoridated toothpaste (Colgate Total Original; Colgate-Palmolive Corp., New York, NY, USA) was applied twice at 12-hour intervals for $3 \mathrm{~min}$ utes to the samples in group 3, followed by cleansing with distilled water. Group 4 underwent application of 0.05\% sodium fluoride (NaF) mouthwash solution for 60 seconds daily. All samples were immersed in artificial saliva and stored in an incubator at $37^{\circ} \mathrm{C}$ between fluoride applications, which were continued for 4 weeks.

\section{Micro-CT scanning and analysis}

Micro-CT was acquired after demineralization (T0), and 2 weeks (T1) and 4 weeks (T2) after fluoridation. The volume of interest for the micro-CT scan was 300 $\mu \mathrm{m}$ (thickness) $\times 300 \mu \mathrm{m}$ (width) $\times 300 \mu \mathrm{m}$ (depth), and a $0.2-\mathrm{mm}$ hole was created with a diamond bur on the $\mathrm{DE}$ and SE to ensure standardization of the scanning site. The samples were scanned with a micro-CT device (Skyscan 1173 ver. 1.6; Bruker-CT, Antwerp, Belgium), which was set at $110 \mathrm{kV}, 72 \mu \mathrm{A}$, and $360^{\circ}$ rotation with $0.15^{\circ}$ steps, to measure the MD at various depths of enamel. The image resolution was 2,240 × 2,240 pixels with a voxel size $9.94 \mu \mathrm{m}$. Two reference phantoms (0.25 $\mathrm{gHAp} / \mathrm{cm}^{3}$ and $0.75 \mathrm{gHAp} / \mathrm{cm}^{3}$ hydroxyapaptite disks) were obtained and scanned under the same conditions as the samples for MD calibration. A $0.3 \mathrm{Al} / 0.25 \mathrm{Cu}$ filter was used to reduce the beam hardening artifacts of micro-CT.

The MD was calculated from the acquired image files (16-bit TIFF) using a three-dimensional (3D) cone beam reconstruction program (NRecon software v1.6.10, SkyScan; Bruker-CT) and analyzed using the CTAn imaging software (v1.9.2, SkyScan; Bruker-CT).

\section{Statistical analysis}

Multivariate analysis of variance was performed using the SPSS software (ver. 23.0; IBM Corp., Armonk, NY, USA) to evaluate the changes in the MD at different time-intervals and among the four groups, followed by Tukey's post hoc test at a significance level of $<0.05$.

\section{RESULTS}

Group 2 showed the highest remineralization of the $\mathrm{DE}$ at the T1-T0 interval (among the four groups) ( $p$ $<0.05$ ), followed by groups 3, 4 and 1 , respectively. Groups 2 and 3 showed significantly higher remineralization during T1-T0 than that at T2-T1 $(p<0.05)$ and exhibited significant changes in the MD after 4 weeks of fluoridation $(p<0.05)$. Group 4 exhibited a relatively consistent increase in the MD over 4 weeks, with no differences in the changes in the MD between T1-T0 and $\mathrm{T} 2-\mathrm{T} 1$; however, the overall MD was significantly in- 
creased ( $p<0.05$, Table 1).

Micro-CT images portraying the MDs exhibited grayscale changes, which were more distinct between T0 and $\mathrm{T} 1$ than those between $\mathrm{T} 1$ and T2 (Figure 1).

Figure 2 shows the linear graphs of the mean MD (vol\%) of each group according to the depths of the enamel, and also between the SE and time-interval within each group. All treatment groups showed profound increments in the MD from T0 to T1 and at T2, albeit to a lesser extent. The total remineralization changes according to the depth of enamel in each group over the course of 4-week fluoridation procedure are shown in Figure 3.

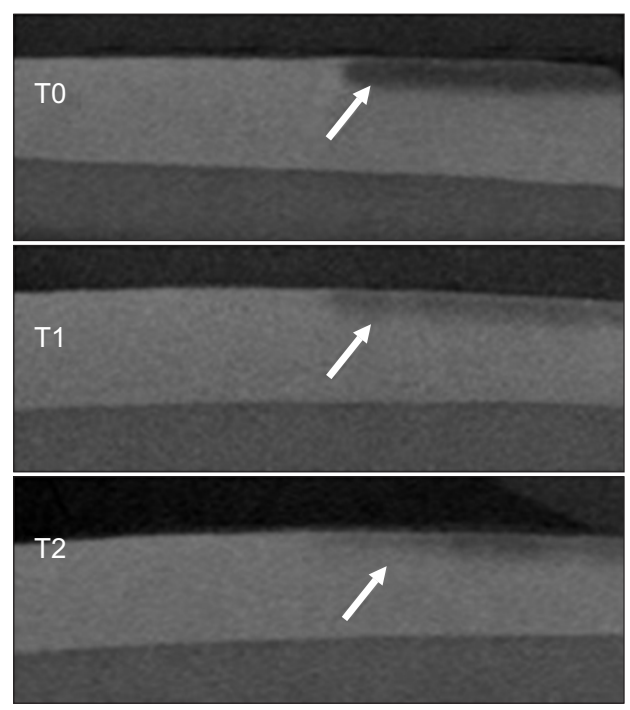

Figure 1. Two dimensional micro-computed tomography images. The arrows indicate mineral density changes as depicted by the gray scale at T0, after demineralization; and T1, 2 weeks and T2, 4 weeks of fluoridation.

\section{DISCUSSION}

The present study demonstrated the remineralization effects of fluoridation with APF gel, toothpaste, and mouthwash regimens on artificially induced WSLs. Micro-CT evaluation revealed that all types of topical fluoride induced an increase in the MD of DE to varying degrees after 4 weeks of application.

The fluoride uptake by enamel is known to be proportional to the duration of contact. One study reported that APF gel application resulted in an initial high concentration of fluoride on the enamel surface. " The APF gel group in our study exhibited the highest remineralization effect on WSLs, which may suggest that the gel consistency and viscosity of APF enable longer contact with the enamel surface, allowing it to thoroughly penetrate the areas around the bracket.

Despite daily application, the NaF solution group showed the lowest remineralization effect among the treatment groups. Moreover, the overall change in the MD of the toothpaste group was higher but to an insignificant degree compared to $\mathrm{NaF}$ solution. The use of high concentration fluoride is not recommended as it arrests remineralization and demineralization, leading to unwanted staining. However, very low concentrations of fluoride $(<50 \mathrm{ppm})$ were also shown to be ineffective for treating WSLs. ${ }^{12}$ Nonetheless, the daily use of $0.05 \%$ $\mathrm{NaF}$ in combination with fluoride toothpaste increased remineralization effect compared to toothpaste alone. ${ }^{13}$ Thus, the simultaneous use of $\mathrm{NaF}$ solution and fluoride toothpaste may be recommended to enhance remineralization of early carious lesions.

All treatment groups showed a higher remineralization effect during the T1-T0 interval than that during the T2-T1 interval. The reduction in the amount of remineralization over time is concurrent with previous studies that stated that the quantity of inorganic ions that penetrate into the lesion decreases with the accumulation of fluorapatite on the enamel surface. ${ }^{14,15}$

Table 1. Changes in the mineral density $\left(\mathrm{gHAp} / \mathrm{cm}^{3}\right)$ of the demineralized enamel among the groups with respect to the time interval

\begin{tabular}{ccccc}
\hline \multirow{2}{*}{ Interval } & \multicolumn{4}{c}{ Group (n= 12) } \\
\cline { 2 - 5 } & $\mathbf{1}$ (Control) & $\mathbf{2}$ (APF gel) & $\mathbf{3}$ (Toothpaste) & 4 (Mouthrinse) \\
\hline $\mathrm{T} 1-\mathrm{T} 0$ & $0.0129 \pm 0.013^{\mathrm{Aab}}$ & $0.1358 \pm 0.013^{\mathrm{Ba}}$ & $0.0844 \pm 0.013^{\mathrm{Ca}}$ & $0.0461 \pm 0.013^{\mathrm{ACa}}$ \\
$\mathrm{T} 2-\mathrm{T} 1$ & $0.0164 \pm 0.010^{\mathrm{Aa}}$ & $0.0461 \pm 0.010^{\mathrm{Ab}}$ & $0.0293 \pm 0.010^{\mathrm{Ab}}$ & $0.0327 \pm 0.010^{\mathrm{Aa}}$ \\
$\mathrm{T} 2-\mathrm{T} 0$ & $0.0294 \pm 0.011^{\mathrm{Aab}}$ & $0.1820 \pm 0.011^{\mathrm{Bc}}$ & $0.1138 \pm 0.011^{\mathrm{Cc}}$ & $0.0788 \pm 0.011^{\mathrm{Cb}}$ \\
\hline
\end{tabular}

Values are presented as mean \pm standard deviation.

Multivariate analysis of variance and Tukey's post-hoc test were performed.

The different letters indicate significant differences $(p<0.05)$; upper-case letters represent comparison between groups within the time interval, and lower-case letters represent comparison between three time intervals within each group.

APF, acidulated phosphate fluoride; T0, after demineralization; T1, 2 weeks of fluoride application; T2, 4 weeks of fluoride application. 

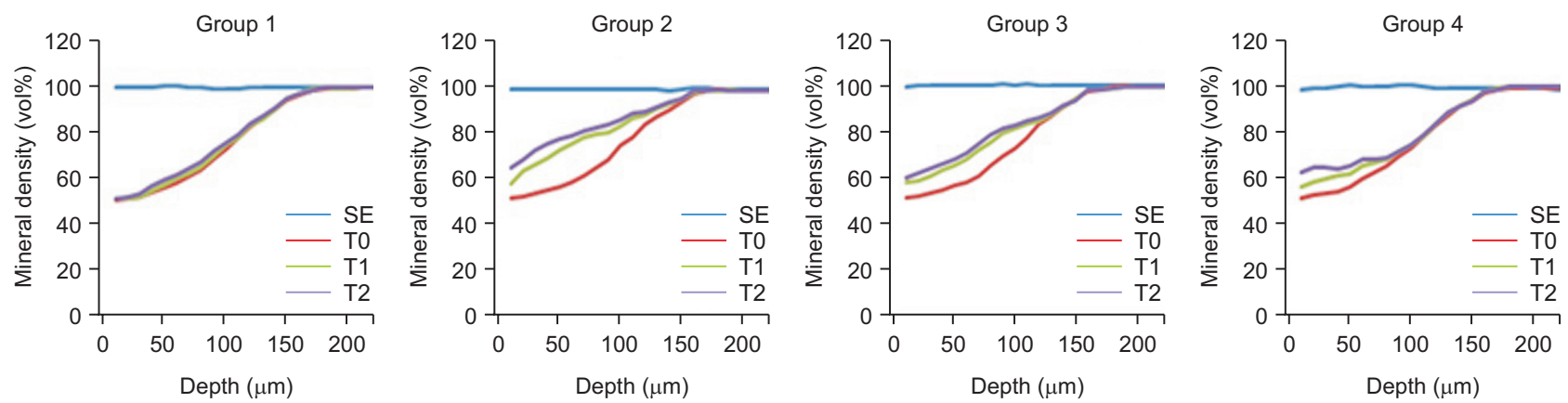

Figure 2. Mean mineral density (vol\%) at various depths of enamel in each group.

Group 1, no treatment; Group 2, 1.23\% acidulated phosphate fluoride gel; Group 3, fluoridated toothpaste; Group 4, $0.05 \%$ sodium fluoride mouthwash solution; SE, sound enamel; T0, after demineralization; T1, 2 weeks of fluoridation; T2, 4 weeks of fluoridation.

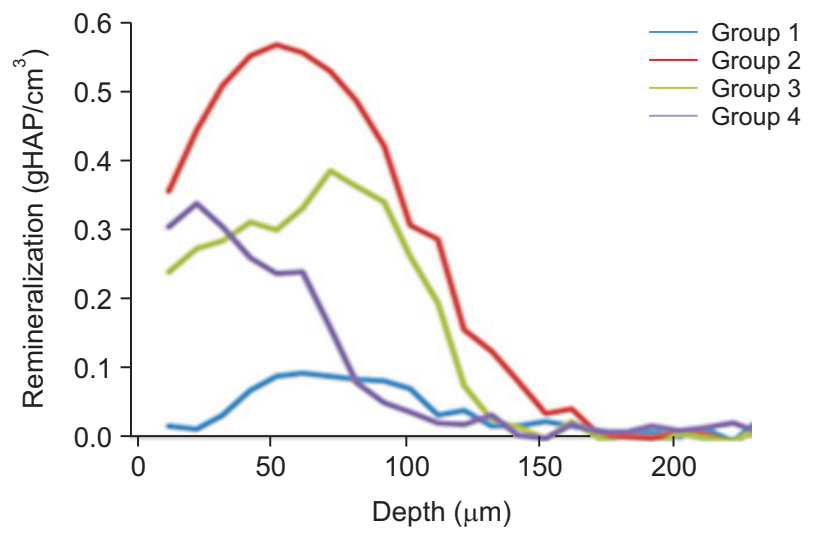

Figure 3. Total remineralization changes in each group over the course of the 4-week fluoridation regimen at various enamel depths.

Group 1, no treatment; Group 2, 1.23\% acidulated phosphate fluoride gel; Group 3, fluoridated toothpaste; Group 4, 0.05\% sodium fluoride mouthwash solution.

Transverse microradiography has been the gold standard for the analysis of the mineral content of dental enamel $;{ }^{16}$ however, it requires sacrifice of the samples, which renders longitudinal analysis of the same sample impossible. In addition to yielding 3D information, micro-CT analysis does not require the destruction of the samples, and thus has a major advantage of providing multiple scans of the same sample over a period of time. $^{17,18}$

The in vitro model is inherently inferior compared to in vivo models as it cannot reproduce the exact conditions and environment of the oral cavity; however, it can provide consistency in terms of experimental conditions. Thus, we were able to obtain a uniform subenamel lesion depth of $150 \mu \mathrm{m}$ with an intact enamel surface and distinct white chalky appearance without the need for air drying. The depth of artificial carious lesions for the purpose of research on remineralization is commonly established within the $50-150 \mu \mathrm{m}$ range. ${ }^{19}$

The in vitro design was among the limitations of this study. Moreover, only three types of separately used fluoride regimens were investigated. Further studies evaluating various combinations of fluoride agents in different forms or comparing fluoridation with other currently available treatment modalities such as CPP-ACP, resin infiltration, and microabrasion are recommended. Attempts to quantify the remineralization effects of different modalities with micro-CT may clarify the treatment guidelines for WSLs.

\section{CONCLUSION}

In this study, all treatment groups showed varying degrees of remineralization of the DE following the 4-week fluoridation protocol. The remineralization effect was higher during the first 2 weeks of application. APF gel was the most effective in inducing remineralization of WSLs from amongst the three fluoride regimens used in this study.

\section{CONFLICTS OF INTEREST}

No potential conflict of interest relevant to this article was reported.

\section{REFERENCES}

1. Gorelick L, Geiger AM, Gwinnett AJ. Incidence of white spot formation after bonding and banding. Am J Orthod 1982;81:93-8.

2. Hadler-Olsen S, Sandvik K, El-Agroudi MA, Øgaard B. The incidence of caries and white spot lesions in orthodontically treated adolescents with a comprehensive caries prophylactic regimen--a prospective study. Eur J Orthod 2012;34:633-9. 
3. Boersma JG, van der Veen MH, Lagerweij MD, Bokhout B, Prahl-Andersen B. Caries prevalence measured with QLF after treatment with fixed orthodontic appliances: influencing factors. Caries Res 2005;39:41-7.

4. Jo SY, Chong HJ, Lee EH, Chang NY, Chae JM, Cho $\mathrm{JH}$, et al. Effects of various toothpastes on remineralization of white spot lesions. Korean J Orthod 2014;44:113-8.

5. Sonesson M, Bergstrand F, Gizani S, Twetman S. Management of post-orthodontic white spot lesions: an updated systematic review. Eur J Orthod 2017;39:116-21.

6. Yetkiner E, Wegehaupt F, Wiegand A, Attin R, Attin T. Colour improvement and stability of white spot lesions following infiltration, micro-abrasion, or fluoride treatments in vitro. Eur J Orthod 2014;36:595602.

7. Bishara SE, Ostby AW. White spot lesions: formation, prevention, and treatment. Semin Orthod $2008 ; 14: 174-82$.

8. Farhadian N, Miresmaeili A, Eslami B, Mehrabi S. Effect of fluoride varnish on enamel demineralization around brackets: an in-vivo study. Am J Orthod Dentofacial Orthop 2008;133(4 Suppl):S95-8.

9. Lee YE, Baek HJ, Choi YH, Jeong SH, Park YD, Song KB. Comparison of remineralization effect of three topical fluoride regimens on enamel initial carious lesions. J Dent 2010;38:166-71.

10. Mielczarek A, Gedrange T, Michalik J. An in vitro evaluation of the effect of fluoride products on white spot lesion remineralization. Am J Dent 2015;28:51-6.

11. Pai N, Mclntyre J, Tadic N, Laparidis C. Compara- tive uptake of fluoride ion into enamel from various topical fluorides in vitro. Aust Dent J 2007;52:41-6.

12. Willmot D. White spot lesions after orthodontic treatment. Semin Orthod 2008;14:209-19.

13. Songsiripradubboon S, Hamba H, Trairatvorakul C, Tagami J. Sodium fluoride mouthrinse used twice daily increased incipient caries lesion remineralization in an in situ model. J Dent 2014;42:271-8.

14. al-Khateeb S, Oliveby A, de Josselin de Jong E, Angmar-Månsson B. Laser fluorescence quantification of remineralisation in situ of incipient enamel lesions: influence of fluoride supplements. Caries Res 1997;31:132-40.

15. ten Cate JM, van de Plassche-Simons YM, van Strijp AJ. Importance of model parameters in the assessment of intra-oral remineralization. J Dent Res 1992;71 Spec No:879-83.

16. Damen JJ, Exterkate RA, ten Cate JM. Reproducibility of TMR for the determination of longitudinal mineral changes in dental hard tissues. Adv Dent Res 1997;11:415-9.

17. Nakata K, Nikaido T, Nakashima S, Nango N, Tagami J. An approach to normalizing micro-CT depth profiles of mineral density for monitoring enamel remineralization progress. Dent Mater J 2012;31:53340.

18. Hamba H, Nikaido T, Sadr A, Nakashima S, Tagami J. Enamel lesion parameter correlations between polychromatic micro-CT and TMR. J Dent Res 2012;91:586-91.

19. White DJ. The application of in vitro models to research on demineralization and remineralization of the teeth. Adv Dent Res 1995;9:175-93; discussion 194-7. 